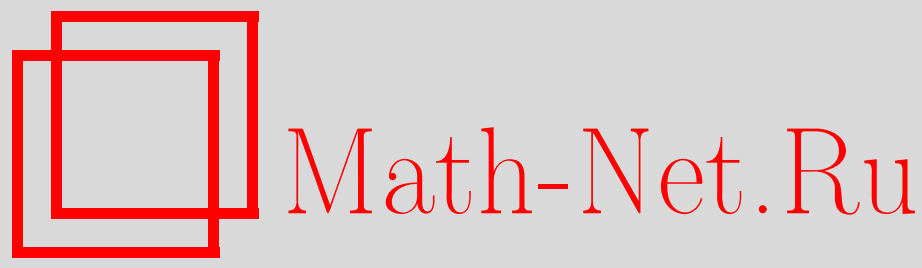

М. Себаве Абдалла, П. Г. Л. Лич, Ли-алгебраический подход к квадратичным инвариантам квантовых систем, ТМФ, 2009, том 159, номер 1, 142-161

DOI: https://doi.org/10.4213/tmf6338

Использование Общероссийского математического портала Math-Net.Ru подразумевает, что вы прочитали и согласны с пользовательским соглашением http://www . mathnet.ru/rus/agreement

Параметры загрузки:

IP: 18.207 .199 .55

26 апреля 2023 г., $17: 20: 41$

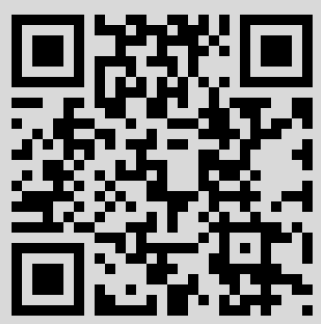




\section{ЛИ-АЛГЕБРАИЧЕСКИЙ ПОДХОД К КВАДРАТИЧНЫМ ИНВАРИАНТАМ КВАНТОВЫХ СИСТЕМ}

Рассмотрена задача о зависящем от времени вырожденном параметрическом усилителе. Получен квадратичный инвариант и на его основе выведена волновая функция, для чего использованы базис в $s u(1,1)$-алгебре и унитарное преобразование к зависящему от времени уравнению Шредингера для параметрического усилителя. Получены вещественные и комплексные инварианты, которые использованы для нахождения решения задачи Коши. С применением различных условий интегрируемости удалось найти наиболее общее решение; приведен подробный анализ этого решения и деталей вычислений.

Ключевые слова: волновая функция, алгебра Ли.

\section{1. ВВЕДЕНИЕ}

Хорошо известно, что задача о вырожденном параметрическом усилителе сыграла важную роль в исследовании различных физических явлений в области квантовой оптики. Гамильтонову модель, описывающую подобную систему, можно построить, используя два или три связанных электромагнитных поля. В оптическом режиме можно наблюдать разделение частот световых пучков как пример параметрического усиления, в котором обе связанные моды являются электромагнитными. Наиболее известный вид параметрического усилителя предназначен для усиления осциллирующего сигнала за счет взаимодействия его частоты и второй, вспомогательной, моды колебаний. В этом случае в результате осцилляции параметра взаимодействия происходит постоянное увеличение энергии как основного сигнала, так и вспомогательной моды. Для такой системы вращательная симметрия в фазовом пространстве состояний основного сигнала оказывается вдвое большей, чем в фазовом пространстве моды накачки. Это фактически означает, что в случае сжатого вакуумного состояния накачка генерирует сигнал с четырехзубцовой структурой [1]. Авторами работы [1] было исследовано влияние накачки некогерентных состояний

* Mathematics Department, College of Science, King Saud University, Riyadh, Saudi Arabia. E-mail: m_sebaweh@physics.org

${ }^{\dagger}$ School of Mathematical Sciences, University of KwaZulu-Natal, Durban, Republic of South Africa. E-mail: leachp@ukzn.ac.za 
на поведение вырожденного параметрического усилителя и определено соотношение между числовыми флуктуациями накачки и сигнала. Сошлемся в этой связи на экспериментальную работу [2], где для возбуждения двухфотонных переходов в атомарном цезии сжатый свет порождался невырожденным параметрическим преобразованием вниз. Интересно также отметить, что влияние сжатой накачки с ненулевой амплитудой было исследовано с помощью параметрической аппроксимации более общего вида, например, в работах [3]-[6]. Наконец, было установлено, что вырожденный параметрический усилитель способен произвести свет, обладающий рядом интересных свойств. Эти свойства могут сохраняться в процессе преобразования частоты, реализуемого устройством, в котором происходит накачка светом с частотой $2 \omega$. Гамильтониан, описывающий такую систему, имеет вид [7]-[12]

$$
\frac{\widehat{H}}{\hbar}=\sum_{i=1}^{2} \omega_{i}\left(\hat{a}_{i}^{\dagger} \hat{a}_{i}+\frac{1}{2}\right)+g\left(\hat{a}_{1}^{\dagger 2} \hat{a}_{2}+\hat{a}_{1}^{2} \hat{a}_{2}^{\dagger}\right),
$$

где $\hat{a}_{1}$ и $\hat{a}_{2}$ - операторы уничтожения для сигнала и накачки соответственно $\left(\left[\hat{a}_{i}, \hat{a}_{j}^{\dagger}\right]=\delta_{i j}\right), \omega_{1}$ и $\omega_{2}$ - частоты сигнала и накачки, а $g$ - параметр взаимодействия. Теперь предположим, что мы пренебрегаем и квантовыми флуктуациями накачки, и ослаблением накачки за счет взаимодействия, так что операторы $\hat{a}_{2}$ и $\hat{a}_{2}^{\dagger}$ можно заменить комплексными числами. Тогда гамильтониан (1.1) сводится к выражению

$$
\frac{\widehat{H}(t)}{\hbar}=\omega\left(\hat{a}^{\dagger} \hat{a}+\frac{1}{2}\right)+\lambda(t)\left(\hat{a}^{\dagger 2} e^{2 i \phi(t)}+\hat{a}^{2} e^{-2 i \phi(t)}\right),
$$

где $\lambda(t)$ - зависящий от времени параметр взаимодействия, в который входит амплитуда классического поля накачки, $\phi(t)$ - зависящая от времени фаза; поскольку теперь имеется всего по одному оператору рождения и уничтожения, мы здесь и далее опускаем индекс у операторов $\hat{a}$ и $\hat{a}^{\dagger}$. Можно видеть, что если определить операторы рождения и уничтожения как

$$
\hat{a}^{\dagger}=(2 \omega \hbar)^{-1 / 2}(\omega \hat{q}-i \hat{p}), \quad \hat{a}=(2 \omega \hbar)^{-1 / 2}(\omega \hat{q}+i \hat{p}),
$$

то указанный гамильтониан примет вид [13]

$$
\widehat{H}(t)=\frac{1}{2} \nu_{(-)}(t) \hat{p}^{2}+\frac{\omega^{2}}{2} \nu_{(+)}(t) \hat{q}^{2}+\mu(t)(\hat{p} \hat{q}+\hat{q} \hat{p}),
$$

где

$$
\nu_{( \pm)}(t)=1 \pm \frac{2 \lambda(t)}{\omega} \cos 2 \phi(t), \quad \mu(t)=\lambda(t) \sin 2 \phi(t) .
$$

Интересно отметить, что зависящий от времени гамильтониан (1.4) отождествляется с зависящим от времени линейным осциллятором, тогда как оператор эволюции взаимодействующей части уравнения (1.2) отождествляется с оператором сжатия одномодового состояния, который применяется в квантовой оптике. Такие задачи подробно рассматривались в последние десятилетия в многочисленных работах (см., например, [14]-[17]). 
Основная цель настояшей статьи такова: используя алгебру Ли группы симметрии, найти наиболее общее решение задачи Коши для уравнения Шредингера

$$
\widehat{H}(t) \psi=i \hbar \frac{\partial \psi}{\partial t}, \quad \psi=\bar{\psi} \quad \text { при } \quad t=0,
$$

не для самого гамильтониана модели (1.4), а для его квадратичного инварианта, поскольку такие решения образуют базис алгебры. Это позволит достичь большей гибкости при получении глобального выражения для волновой функции всей системы [13], [18]--[21]. Сначала мы найдем общее решение уравнений движения в картине Гейзенберга. Это делается в разделе 2. Раздел 3 посвящен рассмотрению волновой функции как в когерентном представлении, так и в квазистационарном представлении числа состояний. Квадратичные инварианты (вещественные и комплексные) рассматриваются в разделе 4. Далее в разделе 5 задача рассматривается на квантовом уровне и на основе алгебр Ли. Наконец, в разделе 6 приведены заключительные замечания, а в приложении обсуждается возможность получения решения задачи в замкнутом виде.

\section{2. УРАВНЕНИЯ ДВИЖЕНИЯ}

Будем искать решения уравнений движения для гамильтониана (1.4) с использованием картины Гейзенберга. Поскольку параметр взаимодействия и фазовая накачка произвольным образом зависят от времени, решение уравнений движения в общем случае зависит от вводимых условий интегрируемости. Действительно, было показано, что использование интегрируемости приводит к нарушению условия некоммутативности гамильтонианов во вращающейся системе отсчета в два различных момента времени, что обычно происходит в зависящей от времени системе, и, следовательно, можно получить решение уравнений движения (см., например, [22]). С другой стороны, с физической точки зрения естественным является предположение, что зависящие от времени фазы и коэффициенты взаимодействия удовлетворяют условию фазового и частотного соответствия. Поэтому удобнее связать зависящее от времени взаимодействие с фазовой накачкой, при этом говорят, что выполнено условие интегрируемости (см., например, [7], [22], [23]).

Уравнения движения в рассматриваемом случае записываются в виде

$$
\frac{d \hat{q}}{d t}=\nu_{(-)}(t) \hat{p}+2 \mu(t) \hat{q}, \quad \frac{d \hat{p}}{d t}=-\omega^{2} \nu_{(+)}(t) \hat{q}-2 \mu(t) \hat{p} .
$$

Как уже отмечалось, трудно ожидать, что можно найти решение этих двух уравнений в замкнутом виде для произвольно зависящих от времени параметра взаимодействия $\lambda(t)$ и фазовой накачки $\phi(t)$. Однако если мы подберем фазовую накачку $\phi(t)$ в форме

$$
\phi(t)=-\omega t+2 \eta I(t)\left(1+\frac{1}{\eta I(t)}\right)^{1 / 2}
$$

или

$$
\phi(t)=-\omega t+2 \eta I(t)
$$


где $\eta$ - произвольная постоянная и $I(t)=\int_{0}^{t} \lambda\left(t^{\prime}\right) d t^{\prime}$, то мы сможем найти зависящие от времени динамические операторы. Следует отметить, что когда параметр $\eta$ попадает в интервал $(-\infty,-1 / 2) \cup(1 / 2, \infty)$, решение соответствует случаю слабого взаимодействия, $\omega>\lambda$, а случай сильной связи получается при $\eta \in[-1 / 2,1 / 2]$. После некоторых вычислений получаем

$$
\begin{aligned}
\hat{q}(t)= & \hat{q}(0)\left[e^{-\theta(t) / 2}\left(\cos \bar{\eta} \theta(t)+\frac{\sin \bar{\eta} \theta(t)}{2 \bar{\eta}}\right) \cos \phi(t)+\right. \\
& \left.+\frac{\eta}{\bar{\eta}} e^{\theta(t) / 2} \sin \bar{\eta} \theta(t) \sin \phi(t)\right]- \\
& -\frac{\hat{p}(0)}{\omega}\left[e^{\theta(t) / 2}\left(\cos \bar{\eta} \theta(t)-\frac{\sin \bar{\eta} \theta(t)}{2 \bar{\eta}}\right) \sin \phi(t)-\right. \\
& \left.-\frac{\eta}{\bar{\eta}} e^{-\theta(t) / 2} \sin \bar{\eta} \theta(t) \cos \phi(t)\right], \\
\hat{p}(t)= & \hat{p}(0)\left[e^{\theta(t) / 2}\left(\cos \bar{\eta} \theta(t)-\frac{\sin \bar{\eta} \theta(t)}{2 \bar{\eta}}\right) \cos \phi(t)+\right. \\
& \left.+\frac{\eta}{\bar{\eta}} e^{-\theta(t) / 2} \sin \bar{\eta} \theta(t) \sin \phi(t)\right]+ \\
& +\omega \hat{q}(0)\left[e^{-\theta(t) / 2}\left(\cos \bar{\eta} \theta(t)+\frac{\sin \bar{\eta} \theta(t)}{2 \bar{\eta}}\right) \sin \phi(t)-\right. \\
& \left.-\frac{\eta}{\bar{\eta}} e^{\theta(t) / 2} \sin \bar{\eta} \theta(t) \cos \phi(t)\right],
\end{aligned}
$$

где мы положили

$$
\bar{\eta}=\sqrt{\eta^{2}-\frac{1}{4}}, \quad \theta(t)=\frac{1}{\eta} \int_{0}^{t} \sqrt{(\omega+\dot{\phi}(t))^{2}-4 \lambda^{2}(t)} d t .
$$

Отметим, что для получения приведенного выше решения мы использовали первый (наиболее общий) вид условия интегрируемости (см. формулу (2.2а)). Альтернативным образом это условие можно записать как

$$
\phi(t)=-\omega t+\eta \operatorname{sh} \theta(t)
$$

Прежде чем двигаться дальше, стоит обратиться к реализации условия интегрируемости (2.2а). Этого можно добиться, если повернуть уравнения движения с помощью гиперболических функций [23]. Условие интегрируемости (2.2б) можно получить и непосредственно на основании соотношения между гамильтонианами в момент времени $t$ и в момент $t_{1}$ в повернутой системе отсчета, если использовать картину Шредингера [22].

Теперь применим динамические операторы, задаваемые уравнениями (2.3) и (2.4), для аккуратного определения операторов рождения (уничтожения) в разные моменты $t_{1}$; далее эти операторы позволят нам диагонализовать гамильтониан (1.4).

6 Теоретическая и математическая физика, т. 159, № 1, 2009 г. 
С этой целью определим оператор

$$
\begin{aligned}
\hat{A}(t)= & \left(\frac{2 \bar{\eta} \omega \hbar}{\eta}\right)^{-1 / 2}\left\{\omega\left[e^{\theta(t) / 2} \cos \phi(t)-\frac{i}{\eta}\left(\bar{\eta}-\frac{i}{2}\right) \sin \phi(t) e^{-\theta(t) / 2}\right] \hat{q}(t)+\right. \\
& \left.+\left[e^{\theta(t) / 2} \sin \phi(t)+\frac{i}{\eta}\left(\bar{\eta}-\frac{i}{2}\right) \cos \phi(t) e^{-\theta(t) / 2}\right] \hat{p}(t)\right\},
\end{aligned}
$$

а также его сопряженный

$$
\begin{aligned}
\hat{A}^{\dagger}(t)= & \left(\frac{2 \bar{\eta} \omega \hbar}{\eta}\right)^{-1 / 2}\left\{\omega\left[e^{\theta(t) / 2} \cos \phi(t)+\frac{i}{\eta}\left(\bar{\eta}+\frac{i}{2}\right) \sin \phi(t) e^{-\theta(t) / 2}\right] \hat{q}(t)+\right. \\
& \left.+\left[e^{\theta(t) / 2} \sin \phi(t)-\frac{i}{\eta}\left(\bar{\eta}+\frac{i}{2}\right) \cos \phi(t) e^{-\theta(t) / 2}\right] \hat{p}(t)\right\} .
\end{aligned}
$$

Оператор $\hat{A}(t)$ и его сопряженный $\hat{A}^{\dagger}(t)$ удовлетворяют коммутационному соотношению $\left[\hat{A}(t), \hat{A}^{\dagger}(t)\right]=\left[\hat{A}(0), \hat{A}^{\dagger}(0)\right]=1$, где $\hat{A}(t)=\hat{A}(0) e^{-i \bar{\eta} \theta(t)}$ и

$$
\hat{A}(0)=\left(\frac{2 \bar{\eta} \omega \hbar}{\eta}\right)^{-1 / 2}\left[\omega \hat{q}(0)+\frac{1}{\eta}\left(\frac{1}{2}+i \bar{\eta}\right) \hat{p}(0)\right] .
$$

Поэтому, подставляя уравнения (2.7) и (2.8) в (1.4), получим

$$
\begin{aligned}
\frac{\widehat{H}}{\hbar}= & \frac{\eta}{\bar{\eta}}(\omega \operatorname{ch} \theta-\lambda \operatorname{sh} \theta) \hat{A}^{\dagger} \hat{A}-\frac{\eta}{4 \bar{\eta}} \hat{A}^{2}\left[(\omega-2 \lambda) e^{\theta}+(\omega+2 \lambda) \xi e^{-\theta}\right]- \\
& -\frac{\eta}{4 \bar{\eta}} \hat{A}^{\dagger 2}\left[(\omega-2 \lambda) e^{\theta}+(\omega+2 \lambda) \xi^{*} e^{-\theta}\right]+\frac{\partial F_{2}}{\partial t}
\end{aligned}
$$

где

$$
\xi=\left(\frac{1}{4 \eta^{2}}-\frac{\bar{\eta}^{2}}{\eta^{2}}-\frac{i \bar{\eta}}{\eta}\right)
$$

a $\partial F_{2} / \partial t$ - частная производная по времени от производящей функции:

$$
\begin{aligned}
\frac{\partial F_{2}}{\partial t}= & \frac{\eta}{\bar{\eta}}\left(\dot{\phi} \operatorname{ch} \theta-\frac{\dot{\theta}}{4 \eta^{2}}\right) \hat{A}^{\dagger} \hat{A}-\frac{\eta}{4 \bar{\eta}} \hat{A}^{2}\left[\dot{\phi}\left(e^{\theta}+\xi e^{-\theta}\right)-\frac{\dot{\theta}}{2 \eta^{2}}(1-2 i \bar{\eta})\right]- \\
& -\frac{\eta}{4 \bar{\eta}} \hat{A}^{\dagger 2}\left[\dot{\phi}\left(e^{\theta}+\xi^{*} e^{-\theta}\right)-\frac{\dot{\theta}}{2 \eta^{2}}(1+2 i \bar{\eta})\right] .
\end{aligned}
$$

Из уравнений (2.10) и (2.12) мы получаем диагонализованный гамильтониан:

$$
\frac{\widehat{H}(t)}{\hbar}=\bar{\eta} \dot{\theta}(t)\left(\hat{A}^{\dagger} \hat{A}+\frac{1}{2}\right) .
$$


Итак, мы показали, что аккуратное определение операторов рождения (уничтожения) приводит к диагонализации гамильтоновой системы. В оставшейся части данного раздела мы рассмотрим случай, когда параметр взаимодействия и фазовая накачка постоянны. При этом квантовые операторы $\hat{q}_{\mathrm{c}}(t)$ и $\hat{p}_{\mathrm{c}}(t)$ записываются как

$$
\begin{aligned}
& \hat{q}_{\mathrm{c}}(t)=\hat{q}_{\mathrm{c}}(0)\left(\cos \Omega_{\mathrm{c}} t+\frac{2 \lambda}{\Omega_{\mathrm{c}}} \sin \Omega_{\mathrm{c}} t \sin 2 \phi\right)+\left(1-\frac{2 \lambda}{\omega} \cos 2 \phi\right) \frac{\sin \Omega_{\mathrm{c}} t}{\Omega_{\mathrm{c}}} \hat{p}_{\mathrm{c}}(0), \\
& \hat{p}_{\mathrm{c}}(t)=\hat{p}_{\mathrm{c}}(0)\left(\cos \Omega_{\mathrm{c}} t-\frac{2 \lambda}{\Omega_{\mathrm{c}}} \sin \Omega_{\mathrm{c}} t \sin 2 \phi\right)-\omega^{2}\left(1+\frac{2 \lambda}{\omega} \cos 2 \phi\right) \frac{\sin \Omega_{\mathrm{c}} t}{\Omega_{\mathrm{c}}} \hat{q}_{\mathrm{c}}(0),
\end{aligned}
$$

где $\Omega_{\mathrm{c}}=\sqrt{\omega^{2}-4 \lambda^{2}}$ и $\omega>2 \lambda$ (случай слабой связи). Интересно сравнить приведенные уравнения с уравнениями (2.3) и (2.4), в которых содержатся зависящие от времени функции. Более точно, усиленная частота $\theta(t)$ содержит все параметры,

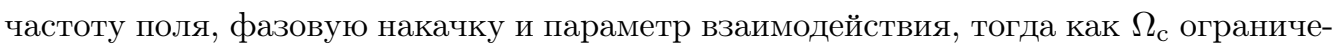
на в том смысле, что содержит не зависящие от времени параметр взаимодействия и частоту поля. На самом деле, тот факт, что в уравнениях (2.3) и (2.4) помимо параметра $\bar{\eta}$ существуют зависящие от времени фазовая накачка и параметр взаимодействия, дает нам широкое многообразие способов контролировать две различные операции: усиление мощности, а также энергетический обмен между полями (где одно из полей является классическим, а другое - квантовым) за счет флуктуаций. Эти две операции определены, когда $\Omega_{c}$ постоянна, в этом случае не существует условия интегрируемости. Таким образом, можно сказать, что наличие зависимости от времени во взаимодействии и в фазе приводит при выполнении условия интегрируемости к возможности управлять некоторыми важными явлениями, такими как параметрический усилитель и преобразователь частоты. Наконец, отметим, что, используя условие интегрируемости (2.2б), можно рассмотреть статистические свойства данной системы, а также возможность физических применений, в которых в качестве начального состояния используется когерентное состояние с добавлением фотона [7].

\section{3. ВОЛНОВАЯ ФУНКЦИЯ}

В данном разделе мы выведем волновую функцию в представлении чисел заполнения по когерентным и по квазистационарным состояниям. Для этого подействуем глауберовым оператором смещения на вакуумное состояние; получим когерентное состояние в виде

$$
\widehat{D}(\alpha, t)|0\rangle=|\alpha, t\rangle=e^{-|\alpha|^{2} / 2} \sum_{n=0}^{\infty} \frac{\alpha^{n}}{\sqrt{n !}}|n, t\rangle .
$$

Если теперь использовать уравнения (2.6) и (3.1), то можно получить волновую функцию в представлении когерентных состояний:

$$
\psi_{\alpha}(q, t)=N(t) \exp \left[-i \frac{\omega}{2 \hbar} F(t) q^{2}+2 i\left(\frac{\bar{\eta} \omega}{\hbar R(t)}\right)^{1 / 2} \alpha(t) e^{-i \chi(t)} q\right]
$$


где $N(t)$ - зависящий от времени нормирующий множитель,

$$
N(t)=\left(\frac{2 \omega \bar{\eta}}{\pi \hbar R(t)}\right)^{1 / 4} \exp \left(-\frac{1}{2}|\alpha|^{2}+\frac{1}{2} \alpha^{2}(t) e^{-2 i \chi(t)}\right),
$$

а параметр когерентности равен $\alpha(t)=\alpha(0) e^{-i \bar{\eta} \theta(t)}$. В уравнении $(3.2)$ использованы обозначения

$$
\begin{aligned}
F(t) & =\frac{2 \eta \operatorname{sh} \theta(t) \sin 2 \phi(t)+\cos 2 \phi(t)-2 i \bar{\eta}}{R(t)} \\
R(t) & =\sin 2 \phi(t)+2 \eta(\operatorname{ch} \theta(t)-\operatorname{sh} \theta(t) \cos 2 \phi(t)), \\
\chi(t) & =\operatorname{arctg}\left(\frac{2 \bar{\eta}}{2 \eta e^{\theta(t)} \operatorname{tg} \phi(t)+1}\right) .
\end{aligned}
$$

Для вывода волновой функции в представлении чисел заполнения по квазистационарным состояниям необходимо решить уравнение Шредингера (1.6), которое мы перепишем как

$$
\left[\nu_{(-)}(t) \frac{\partial^{2}}{\partial q^{2}}-\frac{\omega^{2}}{\hbar^{2}} \nu_{(+)}(t) q^{2}+\frac{2 i}{\hbar} \mu(t)\left(2 q \frac{\partial}{\partial q}+1\right)\right] \psi(q, t)=-\frac{2 i}{\hbar} \frac{\partial}{\partial t} \psi(q, t) .
$$

После некоторых преобразований получаем

$$
\begin{aligned}
\psi_{n}(q, t)= & \left(\frac{2 \omega \bar{\eta}}{\pi \hbar R(t)}\right)^{1 / 4} \frac{\left(e^{i \pi} 2\right)^{-n / 2}}{\sqrt{n !}} H_{n}\left(\sqrt{\frac{2 \omega \bar{\eta}}{\hbar R(t)}} q\right) \times \\
& \times e^{-i \omega F(t) q^{2} / 2 \hbar} e^{-i(n+1 / 2)(\bar{\eta} \theta(t)+\chi(t))} .
\end{aligned}
$$

Можно также вычислить волновую функцию для рассматриваемой системы альтернативным образом, используя условие интегрируемости (2.2б). Получим

$$
\begin{aligned}
\psi_{n}(q, t)= & \left(\frac{\omega \sqrt{\left(\eta^{2}-1\right)}}{\hbar \pi(\eta-\cos 2 \phi(t))}\right)^{1 / 4} \frac{2^{-n / 2}}{\sqrt{n !}} H_{n}\left(\sqrt{\frac{\omega\left(\eta^{2}-1\right)^{1 / 2}}{\hbar(\eta-\cos 2 \phi(t))}} q\right) \times \\
& \times \exp \left(-\frac{\omega}{2 \hbar}\left(\frac{\sqrt{\eta^{2}-1}+i \sin 2 \phi(t)}{\eta-\cos 2 \phi(t)}\right) q^{2}\right) \times \\
& \times \exp \left(-i\left(n+\frac{1}{2}\right)\left[I(t)-\operatorname{arctg}\left(\sqrt{\frac{\eta+1}{\eta-1}} \operatorname{tg} \phi(t)\right)\right]\right) .
\end{aligned}
$$

Нашей следующей задачей является вывод квадратичных инвариантов для гамильтониана (1.4). Этому посвящен следующий раздел.

\section{4. КВАДРАТИЧНЫЕ ИНВАРИАНТЫ}

Хорошо известно, что линейные и квадратичные инварианты для зависящего от времени гамильтониана получены достаточно давно (см., например, [24], [25]). Поскольку мы хотим использовать квадратичные инварианты для нахождения наиболее общей волновой функции с использованием алгебры Ли, мы рассматриваем 
как вещественные, так и комплексные инварианты. Однако интересно отметить, что знаменитый классический квадратичный инвариант Ермакова для одномерного параметрического осциллятора может быть представлен как произведение линейных инвариантов [26]. Знаменитые квадратичные аналоги Льюиса-Ризенфельда для квантового параметрического осциллятора и квантового параметрического циклотронного движения, связанного с многомодовыми параметрическими квантовыми колебаниями, также были представлены как произведения линейных инвариантов. Это позволило описать динамику систем на основе групп Ли [26]. За дальнейшими подробностями мы отсылаем читателя к работам [27]-[29]. Следующий пункт посвящен рассмотрению вещественных инвариантов.

4.1. Вещественные инварианты. Вещественные квадратичные константы движения будем искать в виде [30], [31]

$$
\hat{I}=\beta(t) \hat{p}^{2}+\delta(t) \hat{q}^{2}+\gamma(t)(\hat{q} \hat{p}+\hat{p} \hat{q}),
$$

где $\beta(t), \delta(t)$ и $\gamma(t)$ - зависящие от времени произвольные функции, подлежащие определению. Найдем эти функции, потребовав, чтобы константы движения удовлетворяли соотношению

$$
\frac{d \hat{I}}{d t}=\frac{\partial \hat{I}}{\partial t}+\frac{1}{i \hbar}[\hat{I}, \widehat{H}]=0 .
$$

Из уравнений (1.4) и (4.1), взятых вместе с (4.2), следует, что

$$
\begin{aligned}
\frac{d \beta}{d t}+2 \nu_{(-)}(t) \gamma & =4 \mu(t) \beta, \\
\frac{d \gamma}{d t}+\nu_{(-)}(t) \delta & =\omega^{2} \nu_{(+)}(t) \beta, \\
\frac{d \delta}{d t}+4 \mu(t) \delta & =2 \omega^{2} \nu_{(+)}(t) \gamma .
\end{aligned}
$$

Из этой системы дифференциальных уравнений мы можем получить инвариант. Для этого положим $\beta=\rho^{2}$. Тогда квадратичный инвариант первого рода записывается как

$$
\hat{I}^{(q)}=\left[\frac{c}{\rho^{2}}+\left(\frac{2 \mu \rho-\dot{\rho}}{\nu_{(-)}(t)}\right)^{2}\right] \hat{q}^{2}+\rho^{2} \hat{p}^{2}+\left(\rho \frac{2 \mu \rho-\dot{\rho}}{\nu_{(-)}(t)}\right)(\hat{q} \hat{p}+\hat{p} \hat{q}),
$$

где $\rho(t)$ - решение нелинейного дифференциального уравнения

$$
\begin{gathered}
\ddot{\rho}-\left(\frac{\dot{\nu}_{(-)}(t)}{\nu_{(-)}(t)}\right) \dot{\rho}+\Gamma^{(-)}(t) \rho=\frac{c}{\rho^{3}} \nu_{(-)}^{2}(t), \quad c=\text { const }, \\
\Gamma^{(-)}(t)=\Omega^{2}(t)-2 \nu_{(-)}(t) \frac{d}{d t}\left(\frac{\mu(t)}{\nu_{(-)}(t)}\right), \quad \Omega(t)=\sqrt{\omega^{2}-4 \lambda^{2}(t)} .
\end{gathered}
$$

С другой стороны, если $\delta(t)=\sigma^{2}(t)$, то квадратичный инвариант второго рода принимает вид

$$
\hat{I}^{(p)}=\left(\frac{c}{\sigma^{2}}+\left(\frac{\dot{\sigma}+2 \mu \sigma}{\omega^{2} \nu_{(+)}(t)}\right)^{2}\right) \hat{p}^{2}+\sigma^{2} \hat{q}^{2}+\left(\sigma \frac{\dot{\sigma}+2 \mu \sigma}{\omega^{2} \nu_{(+)}(t)}\right)(\hat{q} \hat{p}+\hat{p} \hat{q}),
$$


где $\sigma(t)$ - решение нелинейного дифференциального уравнения

$$
\begin{gathered}
\ddot{\sigma}-\left(\frac{\dot{\nu}_{(+)}(t)}{\nu_{(+)}(t)}\right) \dot{\sigma}+\Gamma^{(+)}(t) \sigma=\frac{\omega^{4}}{\sigma^{3}} \nu_{(+)}^{2}(t) c, \\
\Gamma^{(+)}(t)=\Omega^{2}(t)+2 \nu_{(+)}(t) \frac{d}{d t}\left(\frac{\mu(t)}{\nu_{(+)}(t)}\right) .
\end{gathered}
$$

В терминах операторов рождения (уничтожения), заданных равенствами (1.3), можно переписать уравнения (4.4) и (4.7) в виде

$$
\begin{aligned}
\frac{\hat{I}^{(q)}}{\hbar}= & \frac{1}{\omega}\left[\frac{c}{\rho^{2}}+\omega^{2} \rho^{2}+\left(\frac{\dot{\rho}-2 \mu \rho}{\nu_{(-)}(t)}\right)^{2}\right]\left(\hat{a}^{\dagger} \hat{a}+\frac{1}{2}\right)+ \\
& +\frac{1}{2 \omega}\left(\frac{c}{\rho^{2}}-\left(\omega \rho+i \frac{\dot{\rho}-2 \mu \rho}{\nu_{(-)}(t)}\right)^{2}\right) \hat{a}^{\dagger 2}+ \\
& +\frac{1}{2 \omega}\left(\frac{c}{\rho^{2}}-\left(\omega \rho-i \frac{\dot{\rho}-2 \mu \rho}{\nu_{(-)}(t)}\right)^{2}\right) \hat{a}^{2}, \\
\frac{\hat{I}^{(p)}}{\hbar}=\omega & {\left[\frac{c}{\sigma^{2}}+\frac{\sigma^{2}}{\omega^{2}}+\left(\frac{\dot{\sigma}+2 \mu \sigma}{\omega^{2} \nu_{(+)}(t)}\right)^{2}\right]\left(\hat{a}^{\dagger} \hat{a}+\frac{1}{2}\right)-} \\
& -\frac{1}{2 \omega}\left(\frac{\omega^{2} c}{\sigma^{2}}-\left(\sigma+i \frac{\dot{\sigma}+2 \mu \sigma}{\omega \nu_{(+)}(t)}\right)^{2}\right) \hat{a}^{\dagger 2}- \\
& -\frac{1}{2 \omega}\left(\frac{\omega^{2} c}{\sigma^{2}}-\left(\sigma-i \frac{\dot{\sigma}+2 \mu \sigma}{\omega \nu_{(+)}(t)}\right)^{2}\right) \hat{a}^{2} .
\end{aligned}
$$

В следующем пункте мы получим комплексный инвариант путем прямого использования уравнения (1.2).

4.2. Комплексный инвариант. Чтобы вывести комплексную квадратичную константу движения, положим

$$
\hat{I}=\bar{\alpha}(t)\left(\hat{a}^{\dagger} \hat{a}+\frac{1}{2}\right)+\bar{\beta}(t) \hat{a}^{\dagger 2}+\bar{\beta}^{*}(t) \hat{a}^{2},
$$

где $\bar{\alpha}(t)$ и $\bar{\beta}(t)$ - соответственно вещественная и комплексная функции. Если использовать уравнения (4.2) и (4.12) вместе с (1.2), получим

$$
\begin{aligned}
\frac{d}{d t} \bar{\alpha}(t) & =4 i \lambda(t)\left[\bar{\beta}^{*}(t) e^{2 i \phi(t)}-\bar{\beta}(t) e^{-2 i \phi(t)}\right], \\
\frac{d}{d t} \bar{\beta}(t) & =2 i \lambda(t) e^{2 i \phi(t)} \bar{\alpha}(t)-2 i \omega \bar{\beta}(t), \\
\frac{d}{d t} \bar{\beta}^{*}(t) & =-2 i \lambda(t) e^{-2 i \phi(t)} \bar{\alpha}(t)+2 i \omega \bar{\beta}^{*}(t) .
\end{aligned}
$$

Отсюда после непосредственных вычислений получаем следующие решения. 
Для условия интегрируемости (2.2а) имеем

$$
\begin{aligned}
2 \bar{\beta}(t)= & \bar{\beta}(0) e^{2 i[\phi(t)-\phi(0)]}\left\{\left[\left(\frac{\eta^{2}}{\bar{\eta}^{2}}+\operatorname{ch} \theta\right) \cos 2 \bar{\eta} \theta-\frac{1}{2 \bar{\eta}}\left(\sin 2 \bar{\eta} \theta \operatorname{sh} \theta+\frac{1}{2 \bar{\eta}}\right)\right]-\right. \\
& \left.-i \frac{\eta}{\bar{\eta}}\left[(1+\operatorname{ch} \theta) \sin 2 \bar{\eta} \theta-\frac{1}{\bar{\eta}} \sin ^{2} \bar{\eta} \theta \operatorname{sh} \theta\right]\right\}- \\
& -\bar{\beta}^{*}(0) e^{2 i[\phi(t)+\phi(0)]}\left\{\left[\left(\frac{\eta^{2}}{\bar{\eta}^{2}}-\operatorname{ch} \theta\right) \cos 2 \bar{\eta} \theta+\frac{1}{2 \bar{\eta}}\left(\sin 2 \bar{\eta} \theta \operatorname{sh} \theta-\frac{1}{2 \bar{\eta}}\right)\right]+\right. \\
& \left.+i \frac{\eta}{\bar{\eta}}\left[(1-\operatorname{ch} \theta) \sin 2 \bar{\eta} \theta+\frac{1}{\bar{\eta}} \sin ^{2} \bar{\eta} \theta \operatorname{sh} \theta\right]\right\}+ \\
& +\bar{\alpha}(0) e^{2 i[\phi(t)]}\left[\left(\eta^{2}-\frac{1}{4} \sin 2 \bar{\eta} \theta\right) \frac{\operatorname{sh} \theta}{\bar{\eta}}-\right. \\
& \left.-\frac{1}{2}(1-\operatorname{ch} \theta \cos 2 \bar{\eta} \theta)+i \frac{\eta}{\bar{\eta}} \sin ^{2} \bar{\eta} \theta\right]
\end{aligned}
$$

Используя соотношение

$$
|\bar{\beta}(t)|^{2}=\frac{1}{4} \bar{\alpha}^{2}(t)+C_{1}, \quad C_{1}=\text { const },
$$

из приведенного уравнения и его комплексно сопряженного можно получить выражение для вещественного параметра $\bar{\alpha}(t)$.

Для условия интегрируемости (2.2б) получаем

$$
\begin{aligned}
2 \bar{\beta}(t)= & \bar{\beta}(0) e^{2 i[\phi(t)-\phi(0)]}\left[\left(\frac{\eta^{2}}{\kappa^{2}}+1\right) \cos 4 \kappa I(t)-\frac{1}{\kappa^{2}}\right]+ \\
& +\bar{\beta}^{*}(0) e^{2 i[\phi(t)+\phi(0)]}\left[\left(\frac{\eta^{2}}{\kappa^{2}}-1\right) \cos 4 \kappa I(t)-2 i \frac{\eta}{\kappa} \sin 4 \kappa I(t)-\frac{1}{\kappa^{2}}\right]+ \\
& +\bar{\alpha}(0) e^{2 i[\phi(t)]}\left[\frac{2 \eta}{\kappa^{2}} \sin ^{2} 2 \kappa I(t)+\frac{i}{\kappa} \sin 4 \kappa I(t)\right]
\end{aligned}
$$

где $\kappa=\sqrt{\eta^{2}-1}$. Как и прежде, при нахождении выражения для $\bar{\alpha}(t)$ мы использовали (4.15).

\section{5. КВАНТОВЫЙ ПОДХОД И ПОДХОД \\ НА ОСНОВЕ АЛГЕБРЫ ЛИ}

В данном разделе мы сконцентрируемся на нахождении наиболее общего решения задачи Коши для уравнения Шредингера (1.6) с помощью метода, основанного на алгебре Ли группы симметрии квадратичного инварианта [32]. Метод, который мы применяем, можно в общих чертах описать следующим образом: при заданной группе Ли симметрий константы движения можно выбрать подходящий базис для алгебры Ли этой группы, при этом группа Ли является также подобной группой симметрий для дифференциального уравнения в частных производных инвариантной системы [33], [34]. Поскольку группа Ли изоморфна матричной группе Ли $\mathcal{G}^{\prime}$, 
мы используем матричную реализацию группы $\mathcal{G}$ для записи образа дифференциального уравнения в частных производных в $\mathcal{G}^{\prime}$. Затем мы решаем дифференциальное уравнение в частных производных в матричном виде, что дает выражения для однопараметрической подгруппы, связанной с базисными элементами алгебры Ли группы $\mathcal{G}$. Таким образом, изоморфизм между $\mathcal{G}$ и $\mathcal{G}^{\prime}$ позволяет получить решение дифференциального уравнения в частных производных в желаемом представлении. Но сначала мы рассмотрим задачу с квантовой точки зрения путем получения волновой функции для каждого инварианта.

5.1. Квантовый подход. Рассмотрим квадратичный инвариант $\hat{I}^{(q)}$, задаваемый уравнением (4.4). Если определить оператор уничтожения

$$
\hat{\mathbf{A}}(t)=\left(2 \hbar c^{1 / 2}\right)^{-1 / 2}\left[\left(\frac{c^{1 / 2}}{\rho}+i \frac{\dot{\rho}-2 \mu \rho}{\nu_{(-)}(t)}\right) \hat{q}+i \rho \hat{p}\right]
$$

где $\left[\hat{\mathbf{A}}, \hat{\mathbf{A}}^{\dagger}\right]=1$, то мы можем использовать представление когерентных состояний (3.1) для нахождения волновой функции в представлении числа состояний в виде

$$
\psi_{n}(q, t)=\left(\frac{c^{1 / 2}}{\pi \hbar \rho^{2}}\right)^{1 / 4} \frac{2^{-n / 2}}{\sqrt{n !}} H_{n}\left(\frac{c^{1 / 4}}{\sqrt{\hbar} \rho} q\right) \exp \left[-\frac{1}{2 \hbar}\left(\frac{c^{1 / 2}}{\rho^{2}}+i \frac{\dot{\rho} / \rho-2 \mu}{\nu_{(-)}(t)}\right) q^{2}\right] .
$$

Следует заметить, что соотношение между квадратичным инвариантом $\hat{I}^{(q)}$ и оператором уничтожения $\hat{A}$ имеет вид

$$
\hat{I}^{(q)}=2 \hbar c^{1 / 2}\left(\hat{\mathbf{A}}^{\dagger} \hat{\mathbf{A}}+\frac{1}{2}\right) .
$$

Аналогично можно определить другой оператор, соответствующий квадратичному инварианту $\hat{I}^{(p)}$, заданному в $(4.7)$ :

$$
\widehat{\mathbf{B}}(t)=\left(2 \hbar c^{1 / 2}\right)^{-1 / 2}\left[\left(\frac{c^{1 / 2}}{\sigma}+i \frac{\dot{\sigma}+2 \mu \sigma}{\omega^{2} \nu_{(+)}(t)}\right) \hat{p}-i \sigma \hat{q}\right],
$$

где $\left[\widehat{\mathbf{B}}, \widehat{\mathbf{B}}^{\dagger}\right]=1$. В этом случае волновая функция в представлении числа состояний принимает вид

$$
\bar{\psi}_{n}(p, t)=\left(\frac{c^{1 / 2}}{\pi \hbar \sigma^{2}}\right)^{1 / 4} \frac{2^{-n / 2}}{\sqrt{n !}} H_{n}\left(\frac{c^{1 / 4}}{\sqrt{\hbar} \sigma} p\right) \exp \left[-\frac{1}{2 \hbar}\left(\frac{c^{1 / 2}}{\sigma^{2}}+i \frac{\dot{\sigma} / \sigma+2 \mu}{\omega^{2} \nu_{(+)}(t)}\right) p^{2}\right]
$$

а соотношение между квадратичным инвариантом $\hat{I}^{(p)}$ и оператором уничтожения $\widehat{\mathbf{B}}$ записывается как

$$
\hat{I}^{(p)}=2 \hbar c^{1 / 2}\left(\widehat{\mathbf{B}}^{\dagger} \widehat{\mathbf{B}}+\frac{1}{2}\right) .
$$

Теперь рассмотрим нашу задачу с точки зрения алгебры Ли. Это делается в следующем пункте. 
5.2. Ли-алгебраический подход. Чтобы проанализировать задачу с точки зрения алгебр Ли, начнем с введения операторов $\widehat{K}_{ \pm}$и $\widehat{K}_{0}$, которые удовлетворяют коммутационным соотношениям

$$
\left[\widehat{K}_{-}, \widehat{K}_{+}\right]=2 \widehat{K}_{0}, \quad\left[\widehat{K}_{0}, \widehat{K}_{ \pm}\right]= \pm \widehat{K}_{ \pm}
$$

Эти операторы являются генераторами алгебры Ли $s u(1,1)$; их можно выразить в терминах бозонных операторов уничтожения и рождения $\hat{a}, \hat{a}^{\dagger}$ :

$$
\widehat{K}_{+}=\frac{1}{2} \hat{a}^{\dagger 2}, \quad \widehat{K}_{-}=\frac{1}{2} \hat{a}^{2}, \quad \widehat{K}_{0}=\frac{1}{2}\left(\hat{a}^{\dagger} \hat{a}+\frac{1}{2}\right),
$$

где $\hat{a}$ и $\hat{a}^{\dagger}$ удовлетворяют обычному коммутационному соотношению $\left[\hat{a}, \hat{a}^{\dagger}\right]=1$. Можно также видеть, что оператор Казимира $\widehat{K}$ для генераторов $\widehat{K}_{ \pm}$и $\widehat{K}_{0}$ удовлетворяет соотношению

$$
\widehat{K}^{2}=\widehat{K}_{0}^{2}-\left(\widehat{K}_{-} \widehat{K}_{+}+\widehat{K}_{+} \widehat{K}_{-}\right)
$$

и, кроме того, $\left[\widehat{K}^{2}, \widehat{K}_{ \pm}\right]=\left[\widehat{K}^{2}, \widehat{K}_{0}\right]=0$. Эти генераторы также имеют следующие свойства:

$$
\begin{gathered}
\widehat{K}^{2}|m ; k\rangle=k(k-1)|m ; k\rangle, \quad \widehat{K}_{0}|m ; k\rangle=(m+k)|m ; k\rangle, \\
\widehat{K}_{+}|m ; k\rangle=[(m+1)(m+2 k)]^{1 / 2}|m+1 ; k\rangle, \\
\widehat{K}_{-}|m ; k\rangle=[m(m+2 k-1)]^{1 / 2}|m-1 ; k\rangle,
\end{gathered}
$$

на основании которых мы тоже можем рассматривать статистические свойства для данной системы, хотя и с иной точки зрения. В формулах (5.10) $k$ - индекс Баргмана, а $m$ - любое неотрицательное целое число. Здесь можно отметить, что обобщенные когерентные состояния Барута-Жирарделло, которые являются собственными состояниями понижающих и повышающих операторов некомпактных групп, реализованы как $s u(1,1)$-алгебра Ли [35]. Подробнее задача об $s u(1,1)$-алгебре Ли рассмотрена в работе [36]. Далее мы опишем процедуру решения задачи Коши для квадратичного инварианта или для любой подобной системы, которая содержит генераторы, удовлетворяющие указанным выше свойствам. Для этого можно записать квадратичную систему или, скажем, гамильтониан в виде

$$
\frac{\widehat{H}(t)}{\hbar}=2 F(t) \widehat{K}_{0}+2\left(\widehat{K}_{+} \Lambda^{*}(t)+\widehat{K}_{-} \Lambda(t)\right),
$$

где $F(t)$ и $\Lambda(t)$ - зависящие от времени функции. Чтобы разобраться в задаче Коши, нам требуется решить дифференциальные задачи

$$
\begin{aligned}
\widehat{K}_{0} \Psi_{0} & =i \hbar \frac{\partial \Psi_{0}}{\partial t}, & \Psi_{0}(q, 0) & =\bar{\Psi}_{0}(q), \\
\widehat{K}_{+} \Psi_{+} & =i \hbar \frac{\partial \Psi_{+}}{\partial t}, & \Psi_{+}(q, 0) & =\bar{\Psi}_{+}(q), \\
\widehat{K}_{-} \Psi_{-} & =i \hbar \frac{\partial \Psi_{-}}{\partial t}, & \Psi_{-}(q, 0) & =\bar{\Psi}_{-}(q) .
\end{aligned}
$$


Используя уравнения (1.3) вместе с уравнением (5.8), мы можем привести первое дифференциальное уравнение к виду

$$
\frac{\partial^{2} \Psi_{0}}{\partial q^{2}}-\frac{\omega^{2}}{\hbar^{2}} q^{2} \Psi_{0}=-4 i \omega \frac{\partial \Psi_{0}}{\partial t}
$$

тогда его общее решение таково:

$$
\Psi_{0}(q, t)=\sum_{n=0}^{\infty} C_{n} H_{n}\left(\sqrt{\frac{\omega}{\hbar}} q\right) e^{-\omega q^{2} / 2 \hbar-i(n+1 / 2) t / 2},
$$

где $C_{n}-$ коэффициент Фурье,

$$
C_{n}=\sqrt{\frac{\omega}{\hbar \pi}} 2^{-n}(n !)^{-1} \int_{-\infty}^{\infty} \bar{\Psi}_{0}(q) H_{n}\left(\sqrt{\frac{\omega}{\hbar}} q\right) e^{-\omega q^{2} / 2 \hbar} d q .
$$

Аналогично, для второго дифференциального уравнения в (5.12) имеем

$$
\frac{\partial^{2} \Psi_{+}}{\partial q^{2}}+\frac{\omega^{2}}{\hbar^{2}} q^{2} \Psi_{+}-\frac{\omega}{\hbar}\left(2 q \frac{\partial \Psi_{+}}{\partial q}+\Psi_{+}\right)=4 i \omega \frac{\partial \Psi_{+}}{\partial t},
$$

соответствующее общее решение

$$
\Psi_{+}(q, t)=\sum_{m=-\infty}^{\infty} C_{m} e^{\omega q^{2} / 2 \hbar+i \sqrt{\omega} m q+i m^{2} t / 4}
$$

а константа $C_{m}$ теперь равна

$$
C_{m}=\frac{\sqrt{\omega}}{2 \pi} \int_{-\pi / \sqrt{\omega}}^{\pi / \sqrt{\omega}} \bar{\Psi}_{+}(q) e^{-\omega q^{2} / 2 \hbar+i \sqrt{\omega} m q} d q .
$$

Наконец, третье дифференциальное уравнение в (5.12) можно записать в виде

$$
\frac{\partial^{2} \Psi_{-}}{\partial q^{2}}+\frac{\omega^{2}}{\hbar^{2}} q^{2} \Psi_{-}+\frac{\omega}{\hbar}\left(2 q \frac{\partial \Psi_{-}}{\partial q}+\Psi_{-}\right)=4 i \omega \frac{\partial \Psi_{-}}{\partial t},
$$

соответствующее общее решение

$$
\Psi_{-}(q, t)=\sum_{r=-\infty}^{\infty} C_{r} e^{-\omega q^{2} / 2 \hbar+i \sqrt{\omega} r q+i r^{2} t / 4},
$$

где

$$
C_{r}=\frac{\sqrt{\omega}}{2 \pi} \int_{-\pi / \sqrt{\omega}}^{\pi / \sqrt{\omega}} \bar{\Psi}_{-}(q) e^{\omega q^{2} / 2 \hbar+i \sqrt{\omega} r q} d q
$$

Легко заметить, что можно записать генераторы $\widehat{K}_{ \pm}$и $\widehat{K}_{0}$ в матричном виде:

$$
\widehat{K}_{0}=\frac{1}{2}\left(\begin{array}{cc}
1 & 0 \\
0 & -1
\end{array}\right), \quad \widehat{K}_{+}=\left(\begin{array}{ll}
0 & 1 \\
0 & 0
\end{array}\right), \quad \widehat{K}_{-}=\left(\begin{array}{cc}
0 & 0 \\
-1 & 0
\end{array}\right)
$$


Поскольку оператор $\partial / \partial t$ можно представить как

$$
\frac{\partial}{\partial t}=\left(\begin{array}{cc}
-i F(t) & -2 i \Lambda^{*}(t) \\
2 i \Lambda(t) & i F(t)
\end{array}\right)
$$

матричный образ дифференциального уравнения в частных производных имеет вид

$$
\begin{aligned}
\frac{\partial \psi_{1}}{\partial t} & =-i F(t) \psi_{1}-2 i \Lambda^{*}(t) \psi_{2}, \\
\frac{\partial \psi_{2}}{\partial t} & =i F(t) \psi_{2}+2 i \Lambda(t) \psi_{1} .
\end{aligned}
$$

Следует заметить, что, хотя уравнения подобны тем, которые можно получить из гамильтониана (5.11) с использованием уравнений движения Гейзенберга для операторов $\hat{a}^{\dagger}$ и $\hat{a}$, функция $\psi$ имеет свойства, отличные от свойств операторов рождения и уничтожения. Заметим также, что гамильтонов оператор, как и квадратичный инвариант, принадлежит линейно порожденной $\widehat{K}_{0}$ и $\widehat{K}_{ \pm}$алгебре Ли, которая совпадает с алгеброй Ли группы $S L(2)$. Поэтому оператор $\exp \left(-i \hbar^{-1} \int_{0}^{t} H\left(t^{\prime}\right) d t^{\prime}\right)$ можно выразить как

$$
\exp \left(-\frac{i}{\hbar} \int_{0}^{t} H\left(t^{\prime}\right) d t^{\prime}\right)=e^{a_{0}(t) \widehat{K}_{0}} e^{a_{-}(t) \widehat{K}_{-}} e^{a_{+}(t) \widehat{K}_{+}},
$$

где $a_{0}(t)$ и $a_{ \pm}(t)$ - функции, подлежащие определению. Если теперь $K=\left(K_{i j}\right)$ есть $(2 \times 2)$-матрица, представляющая элемент в базисе нашей алгебры Ли, то $e^{K}$ можно записать в замкнутом виде как $(2 \times 2)$-матрицу $\widetilde{K}=\left(\widetilde{K}_{i j}\right)$. Элементы $\widetilde{K}_{i j}$ связаны с элементами $K_{i j}$ формулами

$$
\begin{aligned}
\widetilde{K}_{11} & =\left[\frac{1}{\sqrt{\Delta}}\left(K_{11}-K_{22}\right) \operatorname{sh} \frac{\sqrt{\Delta}}{2}+\operatorname{ch} \frac{\sqrt{\Delta}}{2}\right] e^{\operatorname{Tr} K / 2}, \\
\widetilde{K}_{12} & =\frac{2 K_{12}}{\sqrt{\Delta}} \operatorname{sh} \frac{\sqrt{\Delta}}{2} e^{\operatorname{Tr} K / 2},
\end{aligned}
$$

а другие два элемента, $\widetilde{K}_{21}$ и $\widetilde{K}_{22}$, можно получить взаимной заменой 1 на 2 . Кроме того,

$$
\operatorname{Tr} K=\left(K_{11}+K_{22}\right), \quad \Delta=\left(K_{11}-K_{22}\right)^{2}+4 K_{12} K_{21} .
$$

Поскольку решения дифференциальных уравнений (5.12) могут быть эквивалентным образом переписаны как результат действия однопараметрических подгрупп $e^{-i \widehat{K}_{0} t / \hbar}$ и $e^{-i \widehat{K}_{ \pm} t / \hbar}$ на начальные условия $\bar{\Psi}_{0}$ и $\bar{\Psi}_{ \pm}$соответственно,

$$
\Psi_{0}(q, t)=e^{-i \widehat{K}_{0} t / \hbar} \bar{\Psi}_{0}(q) \quad \text { и } \quad \Psi_{ \pm}(q, t)=e^{-i \widehat{K}_{ \pm} t / \hbar} \bar{\Psi}_{ \pm}(q),
$$

решение задачи Коши принимает вид

$$
\begin{aligned}
\Psi(q, t)= & \sum_{n=0}^{\infty} \sum_{m=-\infty}^{\infty} \sum_{r=-\infty}^{\infty} A_{m n r} e^{-\omega q^{2} / 2 \hbar+i \sqrt{\omega}(m+r) q+n a_{0}(t) / 2} \times \\
& \times e^{-\left[r^{2} a_{-}(t)+m^{2} a_{+}(t)\right] / 4} H_{n}\left(\sqrt{\frac{\omega}{\hbar}} q\right),
\end{aligned}
$$


где $A_{m n r}=C_{m} C_{n} C_{r}$. Теперь нам осталось найти явные выражения для функций $a_{0}(t)$ и $a_{ \pm}(t)$. Этого можно добиться, если найти решения дифференциального уравнения (5.24) для гамильтониана и для квадратичного инварианта. Начнем с гамильтониана (1.2). В данном случае в уравнении $(5.24)$ надо положить $F(t)=\omega$ и $\Lambda(t)=\lambda(t) e^{2 i \phi(t)}$. Опираясь на условие интегрируемости $(2.2 \mathrm{a})$, найдем общее решение уравнения (5.24) и далее $a_{0}(t)$ и $a_{ \pm}(t)$ :

$$
\begin{aligned}
& a_{0}(t)=2 \ln \left[\left(\cos \bar{\eta} \theta-i \frac{\eta}{\bar{\eta}} \sin \bar{\eta} \theta\right) \operatorname{ch} \frac{\theta}{2}-\frac{1}{2 \bar{\eta}} \sin \bar{\eta} \theta \operatorname{sh} \frac{\theta}{2}\right]-i(\omega t-\eta \operatorname{sh} \theta), \\
& a_{-}(t)=\frac{1}{2} \operatorname{sh} \theta\left(\cos 2 \bar{\eta} \theta-i \frac{\eta}{\bar{\eta}} \sin 2 \bar{\eta} \theta\right)-\frac{1}{2 \bar{\eta}} \operatorname{ch} \theta\left(\frac{1}{2} \sin 2 \bar{\eta} \theta-i \frac{\eta}{\bar{\eta}} \sin ^{2} \bar{\eta} \theta\right), \\
& a_{+}(t)=-\frac{\left(2 \bar{\eta} \operatorname{th} \frac{\theta}{2}-\operatorname{tg} \bar{\eta} \theta\right)+2 i \eta \operatorname{tg} \bar{\eta} \theta \operatorname{th} \frac{\theta}{2}}{\left(2 \bar{\eta}-\operatorname{tg} \bar{\eta} \theta \operatorname{th} \frac{\theta}{2}\right)-2 i \eta \operatorname{tg} \bar{\eta} \theta}
\end{aligned}
$$

Эту же процедуру поиска явных выражений для $a_{0}(t)$ и $a_{ \pm}(t)$ можно применить и к квадратичным инвариантам, указав в уравнении (5.24) вид функций $F(t)$ и $\Lambda(t)$, соответствующий тому, какой из инвариантов мы желаем использовать. Сначала рассмотрим квадратичный инвариант $\hat{I}^{(q)}$, задаваемый формулой (4.10). В этом случае функции $F(t)$ и $\Lambda(t)$ имеют вид

$$
F(t)=\frac{2}{\omega}\left[\frac{c}{\rho^{2}}+\omega^{2} \rho^{2}+\left(\frac{\dot{\rho}-2 \mu \rho}{\nu_{(-)}(t)}\right)^{2}\right], \quad \Lambda(t)=\frac{1}{\omega}\left[\frac{c}{\rho^{2}}-\left(\omega \rho-i \frac{\dot{\rho}-2 \mu \rho}{\nu_{(-)}(t)}\right)^{2}\right]
$$

а потому уравнения, которые нам следует решить, таковы:

$$
i \frac{\partial D}{\partial t}=F(t) D+|\Lambda(t)| e^{-i \Theta(t)} D^{*}, \quad-i \frac{\partial D^{*}}{\partial t}=F(t) D^{*}+|\Lambda(t)| e^{i \Theta(t)} D,
$$

где

$$
\Theta(t)=\operatorname{arctg}\left(i \frac{\Lambda^{*}(t)-\Lambda(t)}{\Lambda^{*}(t)+\Lambda(t)}\right)
$$

- зависящая от времени фаза. Если положить $E=e^{i \Theta(t) / 2} D$ и подставить эту функцию в уравнения (5.31), то мы получим

$$
\begin{aligned}
& \frac{d}{d t}\left(E+E^{*}\right)=i\left(F(t)-\frac{\dot{\Theta}(t)}{2}-|\Lambda(t)|\right)\left(E^{*}-E\right), \\
& \frac{d}{d t}\left(E^{*}-E\right)=i\left(F(t)-\frac{\dot{\Theta}(t)}{2}+|\Lambda(t)|\right)\left(E+E^{*}\right) .
\end{aligned}
$$

Трудно ожидать, что мы сможем найти общее решение этих уравнений в замкнутом виде. Однако имеются две возможности для получения общего решения. Одна из них состоит в использовании условия интегрируемости

$$
\left(\frac{\dot{\Theta}(t)}{2}+\zeta|\Lambda(t)|\right)^{2}-|\Lambda(t)|^{2}=4 c
$$


где $\zeta \in(-\infty,-1 / 2) \cup(1 / 2, \infty)$ - произвольная постоянная. В этом случае имеем общее решение

$$
D(t)=\left(\cos 2 \bar{\zeta} J(t)-\frac{i \zeta}{\bar{\zeta}} \sin 2 \bar{\zeta} J(t)\right) e^{-i \Theta_{-}(t)} D(0)-\frac{i}{2 \bar{\zeta}} e^{-i \Theta_{+}(t)} \sin 2 \bar{\zeta} J(t) \cdot D^{*}(0)
$$

где

$$
\bar{\zeta}=\sqrt{\zeta^{2}-\frac{1}{4}}, \quad \Theta_{ \pm}(t)=\Theta(t) \pm \Theta(0), \quad J(t)=\int_{0}^{t}\left|\Lambda\left(t^{\prime}\right)\right| d t^{\prime} .
$$

Отсюда можно получить $a_{0}(t)$ и $a_{ \pm}(t)$ :

$$
\begin{aligned}
& a_{0}(t)=2 \ln \left(\cos 2 \bar{\zeta} J(t)-\frac{i \zeta}{\bar{\zeta}} \sin 2 \bar{\zeta} J(t)\right)-2 i \Theta_{-}(t), \\
& a_{+}(t)=\frac{2 \zeta \sin ^{2} 2 \bar{\zeta} J(t)-i \bar{\zeta} \sin 4 \bar{\zeta} J(t)}{4 \zeta^{2}-\cos ^{2} 2 \bar{\zeta} J(t)}, \\
& a_{-}(t)=-\frac{i}{4 \bar{\zeta}}\left(\sin 4 \bar{\zeta} J(t)-2 \frac{i \zeta}{\bar{\zeta}} \sin ^{2} 2 \bar{\zeta} J(t)\right),
\end{aligned}
$$

чем и завершается поиск решения.

Другая возможность состоит в том, чтобы рассмотреть

$$
\Phi(t)=\operatorname{arcth}\left(\frac{2|\Lambda(t)|}{2 F(t)-\dot{\Theta}(t)}\right), \quad \Upsilon(t)=\sqrt{\left(F(t)-\frac{\dot{\Theta}(t)}{2}\right)^{2}-|\Lambda(t)|^{2}}
$$

и взять $\Upsilon(t)=\varsigma \dot{\Phi}(t)$, где $\varsigma-$ произвольная постоянная. В этом случае имеем

$$
\begin{aligned}
D(t)= & e^{-i \Theta_{-}(t) / 2} D(0)\left[\left(\cos \bar{\zeta} \Phi-\frac{i \varsigma}{\bar{\varsigma}} \sin \bar{\varsigma} \Phi\right) \operatorname{ch} \frac{\Phi}{2}-\frac{1}{2 \bar{\varsigma}} \sin \bar{\zeta} \Phi \operatorname{sh} \frac{\Phi}{2}\right]- \\
& -e^{-i \Theta_{+}(t) / 2} D^{*}(0)\left[\left(\cos \bar{\zeta} \Phi+\frac{i \varsigma}{\bar{\varsigma}} \sin \bar{\zeta} \Phi\right) \operatorname{sh} \frac{\Phi}{2}-\frac{1}{2 \bar{\varsigma}} \sin \bar{\zeta} \Phi \operatorname{ch} \frac{\Phi}{2}\right]
\end{aligned}
$$

где $\bar{\varsigma}=\sqrt{\varsigma^{2}-1 / 4}$ и $|\varsigma|>1 / 2$. Получив явно зависящее от времени решение для $D(t)$, можно найти $a_{0}(t)$ и $a_{ \pm}(t)$, завершая тем самым решение.

\section{6. ЗАКЛЮЧЕНИЕ}

В настоящей работе рассмотрена задача квантовой оптики о вырожденном параметрическом усилителе при наличии некоторых разумных условий. Главное условие состоит в том, что накачка предполагается достаточно большой для того, чтобы квантовыми флуктуациями можно было пренебречь и чтобы они эффективно не ослаблялись за счет накачки. Оказалось, что гамильтониан имеет различные интерпретации в зависимости от его представления: в терминах операторов рождения и уничтожения гамильтониан отождествляется с одномодовым оператором сжатия, тогда как в терминах координаты и сопряженного импульса гамильтониан является гамильтонианом зависящего от времени линейного осциллятора. 
Мы представили в замкнутом виде решения для зависящего от времени линейного осциллятора в картине Гейзенберга в случаях, когда фаза накачки имеет один из двух конкретных видов. Хотя решения в замкнутом виде для зависящих от времени систем с произвольной зависимостью от времени существуют, наше решение можно использовать и для сильной, и для слабой связи в зависимости от значения параметров. Из решения, полученного в картине Гейзенберга мы смогли построить операторы рождения и уничтожения, в терминах которых гамильтониан зависящего от времени линейного осциллятора принимает диагональный вид, и затем получили волновую функцию.

Подход к решению этой задачи на основе алгебры Ли требует существования достаточного числа инвариантов, чтобы обеспечить наличие базиса замкнутой алгебры. В случае одномерного квадратичного гамильтониана должны существовать три инварианта, поскольку тремя способами можно получить линейно независимые квадратичные функции двух независимых переменных. Имеются два стандартных представления для этих алгебр: $s u(1,1) \sim s o(2,1)$ и $\operatorname{sl}(2, \mathbb{R})$. Мы использовали первое из них и решили три соответствующих уравнения Шредингера. Далее мы использовали матричное представление алгебры для того, чтобы выразить экспоненту от оператора зависящего от времени гамильтониана в виде произведения трех экспонент от элементов алгебры. При этом основная задача свелась к поиску зависящих от времени числовых (комплекснозначных) функций в разложении. Имеются и другие алгебраические подходы [37]-[42], но, по нашему мнению, представленный в настоящей работе подход является оригинальным.

\section{ПРИЛОЖЕНИЕ}

Заметим что при произвольных $\lambda(t)$ и $\phi(t)$ найти решение в замкнутом виде для системы (2.1) затруднительно. В этом приложении мы покажем, в чем суть проблемы, путем сведения решения системы (2.1) к решению одного базисного уравнения. Будем искать преобразование, которое делает уравнения (2.1) автономными. Это эквивалентно нахождению зависящего от времени линейного канонического преобразования [43]

$$
\left(\begin{array}{l}
Q \\
P
\end{array}\right)=\left(\begin{array}{ll}
S_{1} & S_{2} \\
S_{3} & S_{4}
\end{array}\right)\left(\begin{array}{l}
q \\
p
\end{array}\right)
$$

где матрица преобразования $S$ является решением уравнения

$$
\dot{S}=S J A-J \bar{A} S
$$

при

$$
J=\left(\begin{array}{cc}
0 & 1 \\
-1 & 0
\end{array}\right), \quad A=\left(\begin{array}{cc}
2 \mu & \nu_{-} \\
-\omega^{2} \nu_{+} & -2 \mu
\end{array}\right), \quad \bar{A}=\rho^{-2}\left(\begin{array}{ll}
1 & 0 \\
0 & 1
\end{array}\right),
$$

и введению нового времени $T=\int \rho^{-2}(t) d t$ для получения обобщенного канонического преобразования [44], [45]. Система четырех обыкновенных дифференциальных 
уравнений первого порядка, определяющая преобразование, имеет вид

$$
\begin{array}{ll}
\dot{S}_{1}=-\omega^{2} \nu_{+} S_{1}-2 \mu S_{2}-\frac{S_{3}}{\rho^{2}}, & \dot{S}_{2}=-2 \mu S_{1}-\nu_{-} S_{2}-\frac{S_{4}}{\rho^{2}}, \\
\dot{S}_{3}=-\omega^{2} \nu_{+} S_{3}-2 \mu S_{4}+\frac{S_{1}}{\rho^{2}}, & \dot{S}_{4}=-2 \mu S_{3}-\nu_{-} S_{4}+\frac{S_{2}}{\rho^{2}} .
\end{array}
$$

Заметим, что $\rho(t)$ пока не задано.

Стандартный метод решения системы (П.4) состоит в том, чтобы положить $S_{2}=0$, в результате чего преобразование принимает вид преобразования Куммера-Лиувилля по $q$ и $t$. Используя этот анзац, можно исключить $S_{3}$ и $S_{4}$ из системы (П.4) и получить линейное обыкновенное дифференциальное уравнение второго порядка

$$
\ddot{S}_{1}+2\left(\frac{\dot{\rho}}{\rho}+\omega^{2} \nu_{+}\right) \dot{S}_{1}+\left[\frac{1}{\rho^{2}}\left(\omega^{2} \nu_{+} \rho^{2}\right)^{\cdot}+\omega^{4} \nu_{+}^{2}+4 \mu^{2}+\frac{1}{\rho^{4}}\right] S_{1}=0
$$

Это уравнение можно привести к нормальной форме с помощью преобразования

$$
S_{1}=\rho^{-1} \exp \left[-\int \omega^{2} \nu_{+} d t\right] y .
$$

Уравнение для $y(t)$, которое при этом возникает, имеет простой вид

$$
\ddot{y}-\left[\frac{\ddot{\rho}}{\rho}-4 \mu^{2}-\frac{1}{\rho^{4}}\right] y=0 .
$$

Если потребовать, чтобы $\rho$ было решением уравнения Ермакова-Пинни [26], [46]

$$
\ddot{\rho}-4 \mu^{2} \rho=\frac{1}{\rho^{3}},
$$

то решение уравнения (П.7) будет иметь вид $y=A+B t$, причем для простоты можно взять $A=1$ и $B=0$. Тогда

$$
\begin{aligned}
S_{1} & =\rho^{-1} \exp \left[-\int \omega^{2} \nu_{+} d t\right], & S_{2} & =0, \\
S_{3} & =\frac{\dot{\rho}}{\rho} S_{1}, & S_{4} & =-2 \mu \rho^{2} S_{1} .
\end{aligned}
$$

Напомним, Пинни показал, что решение уравнения (П.8) дается нелинейной суперпозицией линейно независимых решений уравнения $\ddot{u}-4 \mu^{2} u=0$, так что поиск решения уравнения (2.1) в замкнутом виде для произвольного $\mu(t)$ эквивалентен нахождению в замкнутом виде решения произвольного линейного обыкновенного дифференциального уравнения второго порядка. Однако следует также заметить, что нам предстоит “сражаться” только с этой функцией, а не с функциями $\nu_{+}$или $\nu_{-}$.

Благодарности. М. С. Абдалла благодарен за финансовую поддержку Research Center, College of Science, King Saud University (проект Math/2009/32). П. Г. Л. Лич благодарит Department of Information and Communication Systems Engineering of the University of the Aegean, Greece, за предоставление благоприятных условий для работы; часть настоящей работы была выполнена при поддержке University of KwaZulu-Natal. 


\section{Список литературы}

[1] M. Hillery, D. Yu., J. Bergou, Phys. Rev. A, 49:2 (1994), 1288-1295.

[2] N. Ph. Georgiades, E. S. Polzik, K. Edamatsu, H. J. Kimble, Phys. Rev. Lett., 75:19 (1995), 3426-3429.

[3] C. C. Gerry, J. Opt. Soc. Amer. B, 8:3 (1991), 685-690.

[4] C. C. Gerry, Phys. Rev. A, 35:5 (1987), 2146-2149; C. C. Gerry, E. E. Hach, Phys. Lett. A, 174:3 (1993), 185-189.

[5] I. Jex, V. Bužek, J. Modern Opt., 40:5 (1993), 771-783.

[6] M. Zahler, Y. Ben Aryeh, Phys. Rev. A, 43:11 (1991), 6368-6378.

[7] M.S. Abdalla, A. A. El-Orany Faisal, J. Perina, Nuovo Cimento B, 116:2 (2001), 137-154.

[8] V. Bužek, G. Drobný, Phys. Rev. A, 65:5 (2002), 053410.

[9] A. A. El-Orany Faisel, S. S. Hassan, M. S. Abdalla, J. Optics B, 5 (2003), 396-404.

[10] C. W. Gardiner, C. M. Savage, Optics Comm., 50:3 (1984), 173-178.

[11] A. Karpati, P. Adam, J. Janszky, M. Bertolotti, C. Sibilia, J. Optics B, 2:2 (2000), 133-139.

[12] X.-G. Wang, S.-H. Pan, G.-Z. Yang, European J. Phys., 10:3 (2000), 415-422.

[13] M. S. Abdalla, M. A. Bashir, J. Egyptian Math. Soc., 5:2 (1997), 171-182.

[14] H.-Y. Fan, H. R. Zaidi, J. R. Klauder, Phys. Rev. D, 35:6 (1987), 1831-1834.

[15] H.-Y. Fan, J. VanderLinde, Phys. Rev. A, 39:6 (1989), 2987-2993.

[16] C. F. Lo, Phys. Rev. A, 42:11 (1990), 6752-6755.

[17] M. Xin, W. Rhodes, Phys. Rev. A, 41:9 (1990), 4625-4631.

[18] M. S. Abdalla, M. A. Bashir, Quantum Semiclass. Opt., 10:2 (1998), 415-423.

[19] M. A. Al-Gwaiz, M. S. Abdalla, S. Deshmukh, J. Phys. A, 27:4 (1994), 1275-1282.

[20] M.S. Abdalla, Nuovo Cimento B, 112:11 (1997), 1549-1554.

[21] M. A. Bashir, M. S. Abdalla, Phys. Lett. A, 204:1 (1995), 21-25.

[22] E. Y.C. Lu, Phys. Rev. A, 8:2 (1973), 1053-1061; Nuovo Cimento Lett., 3:14 (1972), $585-589$.

[23] M.S. Abdalla, S. S. Hassan, A.-S.F. Obada, Phys. Rev. A, 34:6 (1986), 4869-4874; M. S. Abdalla, M. M. Nassar, Ann. Phys., 324:3 (2009), 637-669.

[24] H. R. Lewis, W. B. Riesenfeld, J. Math. Phys., 10:8 (1969), 1458-1473.

[25] H. R. Lewis Jr., Phys. Rev. Lett., 18:13 (1967), 510-512; J. Math. Phys., 9:11 (1968), 1976-1986.

[26] В. П. Ермаков, Универ. изв. Киев, 9 (1880), 1-25.

[27] I. A. Malkin, V. I. Man'ko, D. A. Trifonov, Phys. Rev. D, 2:8 (1970), 1371-1385.

[28] I. A. Malkin, V. I. Man'ko, D. A. Trifonov, J. Math. Phys., 14:5 (1973), 576-582.

[29] V. V. Dodonov, V. I. Man'ko, Phys. Rev. A, 20:2 (1979), 550-560.

[30] M. S. Abdalla, P. G. L. Leach, J. Phys. A, 36:49 (2003), 12205-12221.

[31] M. S. Abdalla, P. G. L. Leach, J. Phys. A, 38:4 (2005), 881-893.

[32] S. Steinberg, J. Differential Equations, 26:3 (1977), 404-434.

[33] G. Dattoli, M. Richetta, G. Schettini, A. Torre, J. Math. Phys., 31:12 (1990), 2856-2863.

[34] G. Dattoli, J. C. Gallado, A. Torre, Riv. Nuovo Cimento, 11:11 (1988), 1-79.

[35] A. O. Barut, L. Girardello, Comm. Math. Phys., 21:1 (1971), 41-55.

[36] A. O. Barut, "Unified algebraic construction of representations of compact and non-compact Lie algebras and Lie groups", Mathematical Methods of Theoretical Physics, Proc. IX Boulder Summer Institute for Theoretical Physics (Univ. Colorado, Boulder, Colo., 1966), Lectures in Theoret. Phys., IXA, eds. W. E. Brittin, A. O. Barut, M. Guenin, Gordon and Breach, New York, 1967, 125-171.

[37] V. de Alfaro, S. Fubini, G. Furlan, Nuovo Cimento A, 34:4 (1984), 569-612.

[38] K. Andriopoulos, P. G. L. Leach, J. Phys. A, 38:20 (2005), 4365-4374.

[39] K. Andriopoulos, P. G. L. Leach, J. Nonlinear Math. Phys., 12 Suppl. 1 (2005), 32-42.

[40] P. G. L. Leach, K. Andriopoulos, Phys. Scripta, 77:1 (2008), 015002.

[41] R. L. Lemmer, P. G. L. Leach, Arab. J. Math. Sci., 5:2 (1999), 1-17. 
[42] M. Znojil, P. G. L. Leach, J. Math. Phys., 33:8 (1992), 2785-2794.

[43] P. G. L. Leach, J. Math. Phys., 18:8 (1977), 1608-1611.

[44] J.-R. Burgan, Sur les groupes de transformation en physique mathématique. Application aux fluides de l'éspace des phases et à la mécanique quantique, Thèse, Univ. d'Orléans, Orléans, 1978.

[45] J.-R. Burgan, M. R. Feix, E. Fijalkow, J. Gutierrez, A. Munier, "Utilisation des groupes de transformation pour la résolution des équations aux dérivées partielles", Applied Inverse Problems, Lecture Notes in Phys., 85, ed. P. C. Sabatier, Springer, Berlin, 1978, 248-273.

[46] E. Pinney, Proc. Amer. Math. Soc., 1:5 (1950), 681.

Поступила в редакцию 8.05.2008 